\title{
Permeability Coefficient of Unsaturated Loess and Its Gaseous and Liquid Water Migration Modeling
}

\author{
Ya-lin Nan, ${ }^{1}$ Zai-kun Zhao $\mathbb{D}^{2},{ }^{2}$ Xin Jin $\mathbb{D}^{3},{ }^{3}$ Liang Zhang $\mathbb{D}^{2},{ }^{2}$ Xiao-xuan Zhu $\mathbb{D}^{2}$ \\ and Ze-jun Liu $\mathbb{D}^{2}$ \\ ${ }^{1}$ China Electronic Research Institute of Engineering Investigations and Design, Xi'an 710001, China \\ ${ }^{2}$ College of Civil Engineering, Xi'an University of Architecture and Technology, Xi'an 710055, China \\ ${ }^{3}$ School of Civil and Architecture Engineering, Xi'an Technological University, Xi'an 710021, China \\ Correspondence should be addressed to Zai-kun Zhao; zhaozaikun@xauat.edu.cn
}

Received 9 November 2020; Revised 8 February 2021; Accepted 1 March 2021; Published 15 March 2021

Academic Editor: Junping Ren

Copyright (c) 2021 Ya-lin Nan et al. This is an open access article distributed under the Creative Commons Attribution License, which permits unrestricted use, distribution, and reproduction in any medium, provided the original work is properly cited.

Water migration changes the water distribution of loess and significantly affects the engineering properties of loess. However, because of the characteristics of loess such as water sensitivity and special structure, it is still difficult to understand the water migration trend of loess. The objectives of this study are to investigate the water diffusion coefficient and soil-water characteristic curve (SWCC) of loess, focusing on the effect of temperature and density, and establish a mixed migration equation for gaseous and liquid water to develop a new calculation method of loess water migration. The results show that the density of loess with larger liquid water content has a more significant effect on the diffusion coefficient than that of less liquid water content. Furthermore, the density significantly affects the matric suction of unsaturated loess, while the temperature change at normal temperature has slight effect. Based on the data obtained in this study, the soil water permeability coefficient was obtained. Moreover, considering the characteristics of mixed migration of gas and liquid water in unsaturated loess, an equation was derived for the gas-liquid water transfer in unsaturated loess. The calculation results are consistent with the experimental results of water migration experiment. Based on the calculation results of water migration, the trend of water migration and proportion of gaseous water during migration were analyzed.

\section{Introduction}

Permeability coefficient is important to evaluate the permeability of unsaturated soil. The analysis and calculation of water movement require the measurement of permeability coefficient of unsaturated soil. The permeability coefficient of unsaturated soil has been extensively studied. Jeong et al. [1] studied the effect of rainfall-induced volumetric liquid water content on matric suction. Lemoubou et al. [2] developed an estimation method for unsaturated soil permeability parameters considering the effect of volumetric liquid water content. Zhang et al. [3] studied the effect of instantaneous volumetric liquid water content and saturation on the soil suction of thickened oil sand tailings. Kaewsong et al. [4] studied the effect of suction change caused by the change in saturation degree on the small strain stiffness of unsaturated soil. Tian et al. [5] studied the effect of volumetric liquid water content on the permeability coefficient of unsaturated loess under steady state. Trabelsi et al. [6] studied the suction of clays with different saturations.

Permeability parameters vary with the change in soil types [7-9]. Unsaturated loess, one of the most widely distributed soil types, is a special soil with water sensitivity and macropore structure [10-12]. Zhang et al. [13] reported that the permeability coefficient of unsaturated loess is affected by pore characteristics, and the pore characteristics of loess with different densities and water contents under different pressures were studied. Based on the study of macropore characteristics of unsaturated loess and effect of consolidation pressure on permeability coefficient, Wei et al. [14] assumed that the pore characteristics significantly affect the permeability coefficient, and the size and distribution of pore determine the permeability coefficient. By conducting 
constant-head permeability experiments and mercury injection porosimetry on loess specimens of different dry densities, Wang et al. [15] assumed that there is a threshold hydraulic gradient below which no flow is observed, and there is a critical hydraulic gradient below which the relationship between hydraulic gradient and seepage velocity is nonlinear, which is closely related to pore size distribution. Through theoretical analysis, field test, and software simulation, Wang et al. [16] studied the effect of unsaturated loess permeability coefficient on the grouting effect of collapsible loess stratum. Liu et al. [17] used microstructure models to study air permeability.

Many factors, especially temperature, significantly affect the permeability of unsaturated loess [18-21]. Owing to a large daily variation of temperature in the loess plateau, the temperature difference between the upper and lower layers of shallow soil is large. With climate change, the temperature change of shallow soil is also relatively large. The change in loess matrix suction caused by temperature affects the mechanical properties of loess and water migration. In addition, because water flows through the pore space of unsaturated loess, the scale of pore space determines its permeability, i.e., the permeability coefficient of unsaturated loess is not constant. At present, some studies have been conducted on the seepage parameters of unsaturated loess, but it is still difficult to understand the trend of water permeability. It is essential to study the water permeability parameters of unsaturated loess.

The objectives of this study are to investigate the water diffusion coefficient and soil-water characteristic curve (SWCC) of loess, focusing on the effect of temperature and density, and derive a mixed migration equation for gaseous and liquid water, thus developing a new calculation method for loess water migration. The water diffusion coefficient of unsaturated loess considering density effect was studied at various densities $\left(1.2,1.4,1.5\right.$, and $\left.1.6 \mathrm{~g} / \mathrm{cm}^{3}\right)$. Furthermore, the density and temperature effects of SWCC were evaluated by measuring the matric suction at various densities $(1.2,1.4$, 1.5 , and $\left.1.6 \mathrm{~g} / \mathrm{cm}^{3}\right)$ and temperatures $\left(5,15,25\right.$, and $\left.35^{\circ} \mathrm{C}\right)$. Based on the data of water diffusion coefficient and SWCC, the soil water permeability coefficient was obtained. Moreover, considering the characteristics of mixed migration of gas and liquid water in unsaturated loess, an equation was derived for gas-liquid water transfer in unsaturated loess. The data of water diffusivity and SWCC of loess were substituted into the equation for calculation.

\section{Experimental Study on Water Diffusion Coefficient}

To measure the water diffusivity of unsaturated loess, a water diffusivity experiment was carried out. Disturbed loess was taken from an engineering site in Xi'an at a depth of $5 \mathrm{~m}$ below the surface, and the property parameters of loess are shown in Table 1. First, compacted loess samples of different densities were prepared indoors. Then, a horizontal soil column infiltration was used to test the water diffusivity of unsaturated loess. Figure 1 shows the experimental apparatus for water diffusivity. Markov bottle can supply water
TABle 1: Physical parameters of loess samples.

\begin{tabular}{lccc}
\hline $\begin{array}{l}\text { Plastic } \\
\text { limit }\end{array}$ & Liquid limit & Specific gravity & $\begin{array}{c}\text { Maximum dry density } \\
\left(\mathrm{g} / \mathrm{cm}^{3}\right)\end{array}$ \\
\hline 18.1 & 29.6 & 2.71 & 1.69 \\
\hline
\end{tabular}

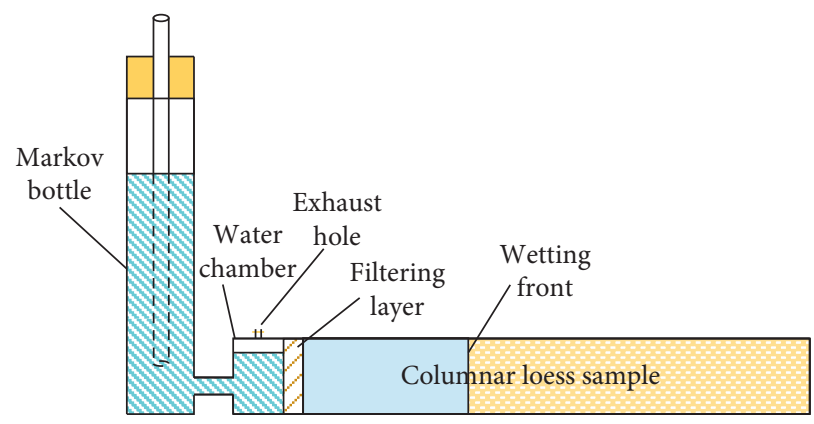

FIGURE 1: Experimental apparatus for water diffusivity.

for the loess samples. The sleeve of columnar loess simple, a plexiglass cylinder with a length of $5 \mathrm{~cm}$ and diameter of $4.2 \mathrm{~cm}$, can be removed from each other to facilitate easy disassembly at the end of experiment to determine the water content. Baffle plates were added at the head and end of soil column and fixed with screws. The total length of soil column is $75 \mathrm{~cm}$. At the inlet boundary, the water chamber with an exhaust hole can keep the water level constant and the soil saturated. A filtering layer with low bubble pressure and high conductivity was installed between the inlet chamber and soil column to ensure that the only driving force in the soil is matrix suction.

First, enough samples (air-dried soil) were prepared, and loess with uniform bulk density was loaded in each cylinder. Second, the screw was tightened, and the soil column was horizontally placed after the soil column was installed. Then, the inlet chamber was instantaneously filled with water, and the exhaust hole was kept open until the air in the inlet chamber was completely exhausted. Subsequently, we start to record the elapsed time and the initial water level reading. Finally, the water supply was stopped, and the fastening screw was loosened after the experiment time. The mass water content of each section of loess was measured by weighing and converted to volumetric water content, and the experiment duration and total water inflow were recorded.

According to the time-dependent water content in the soil column along the length direction, the water diffusion coefficients were obtained using the following equation:

$$
D(\theta)=-\frac{1}{2(\mathrm{~d} \theta / \mathrm{d} \lambda)} \int_{\theta_{0}}^{\theta} \lambda \mathrm{d} \theta
$$

where $D(\theta)$ is the soil water diffusion coefficient $\left(\mathrm{cm}^{2} / \mathrm{min}\right)$; $\lambda=x t^{-(1 / 2)}$ is the transformation parameter; $x$ is the distance from a point in the soil trough to the interface between soil and water tank $(\mathrm{cm}) ; t$ is the test duration $(\mathrm{min}) ; \theta_{0}$ is the uniform initial volume water content of soil column $\left(\mathrm{cm}^{3} / \mathrm{cm}^{3}\right) ; \theta$ is the volume water content of soil column during the diffusion $\left(\mathrm{cm}^{3} / \mathrm{cm}^{3}\right)$. 
Figure 2 expresses the difference in the magnitude of permeability coefficient, and semilog plot was used in the curve, making the original smooth curve wavy. In the logarithmic coordinate, the change in the curve of water diffusion coefficient with volumetric liquid water content is an approximate straight line. Thus, the water diffusion coefficient increases with the increase in volumetric liquid water content. The higher the volumetric liquid water content, the faster the rate of increase of water diffusion coefficient. With the increase in volumetric liquid water content, the water diffusion coefficient of unsaturated loess increases by several orders of magnitude. Besides, the water diffusion coefficient of unsaturated loess increases with the decrease in dry density, and it is more obvious when the dry density is low. Therefore, the effect of density should be fully considered when determining the water diffusion coefficient of loess.

\section{Experimental Study on SWCC of Unsaturated Loess}

To measure the SWCC of unsaturated loess, a matric suction experiment was carried out. The physical properties of soil samples used in the experiment are the same as those of loess used in the water diffusion experiment. The SWCC was measured using a high-speed centrifuge (Hitachi cr21g PF). First, four ring cutters were loaded with the same day density of compacted loess, and those samples were saturated with distilled water. Then, the saturated sample was placed in a centrifuge and dehydrated at a specific speed. Because of the centrifugal force, the water in soil sample will be thrown out into the container below.

The matric-suction-led adsorption of water in soil could be counteracted by centrifugal force. This behavior causes the numerical equality between centrifuge force and suction of soil sample after centrifuge dewatering; that is, the centrifuge force of soil can be regarded as matric suction. Thus, matric suction can be represented by a suction head and calculated from the speed and radius of centrifuge equation (2). The measurement accuracy of matric suction in this study is $1 \mathrm{kPa}$.

By measuring the volume of water thrown out, the volume of residual water in soil sample can be calculated, and the volumetric liquid water content of soil sample can be obtained after the experiment. Finally, by changing the speed of centrifuge, the water content of loess under different suctions can be measured. The final volumetric liquid water content and matric suction are the average values of four samples, and the SWCC of loess with a certain density can be obtained. The above experiments were repeated with loess of other densities. The relationship between volumetric liquid water content and suction head of unsaturated loess with different bulk densities can be obtained.

The suction head $h$ is

$$
h=1.118 r(\mathrm{rpm})^{2},
$$

where $r$ is the centrifugal radius $(\mathrm{cm}), \mathrm{rpm}$ is the rotational speed $(r / \mathrm{min})$, and $h$ is the suction head $(\mathrm{cm})$.

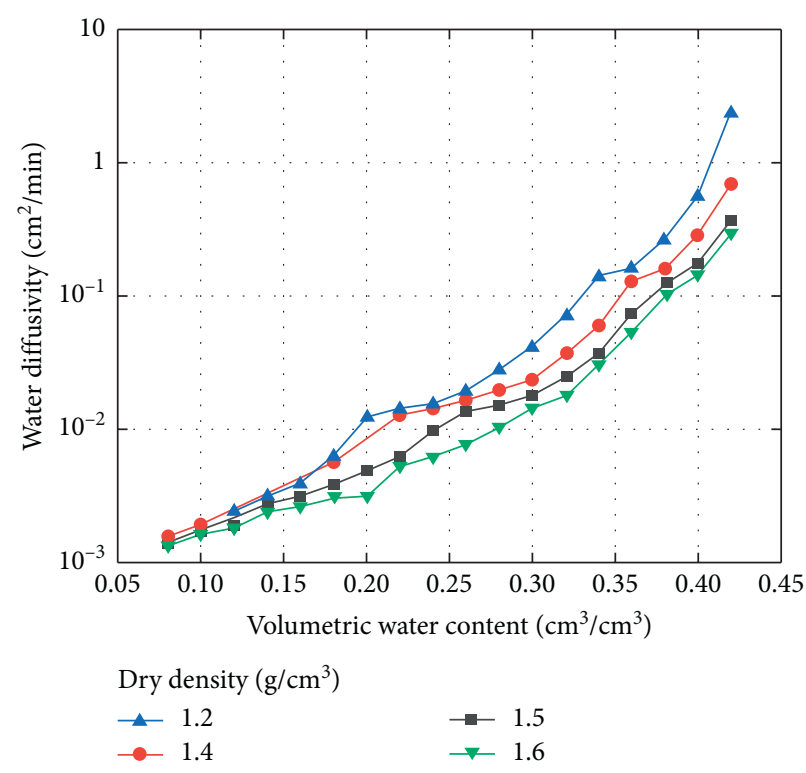

FIgURE 2: Water diffusivity of loess with dry density of 1.2, 1.4, 1.5, and $1.6 \mathrm{~g} / \mathrm{cm}^{3}$ and volumetric liquid water content from 0.08 to $0.42 \mathrm{~cm}^{3} / \mathrm{cm}^{3}$.

Figure 3 shows that temperature has no significant effect on matric suction. For the soil sample with a certain volume moisture content, the suction change caused by temperature change has a certain value. This is small at a high water content and can be ignored but is larger at a low water content. The larger the temperature difference, the greater the change of suction. When studying the water migration of unsaturated soil, the difference in moisture content at different temperatures is not significant when the suction is the same. Therefore, the effect of temperature on suction can be ignored.

Figure 4 shows that when the soil density ratio is small, the change in suction with the density of unsaturated loess at different volumetric liquid water contents is small. When the soil density is large, the change in suction with the density of unsaturated loess at different volumetric liquid water contents is relatively large. The effect of density on matric suction is very significant, mainly caused by the change in density, pore size of soil, and curvature radius of membrane water change.

\section{Calculation Method for Permeability Coefficient of Unsaturated Loess}

First, the parameters of SWCC model were analyzed and determined. Soil density significantly affects the matric suction of unsaturated loess; therefore, the density factor must be considered when fitting the equation. Although temperature can hardly affect the matric suction of unsaturated loess, the temperature factor can be ignored in the fitting equation.

The models used to represent SWCC are usually the Gardner model [22], VG model [23], and Fredlund model [24]. In this study, the SWCC is represented by VG model: 


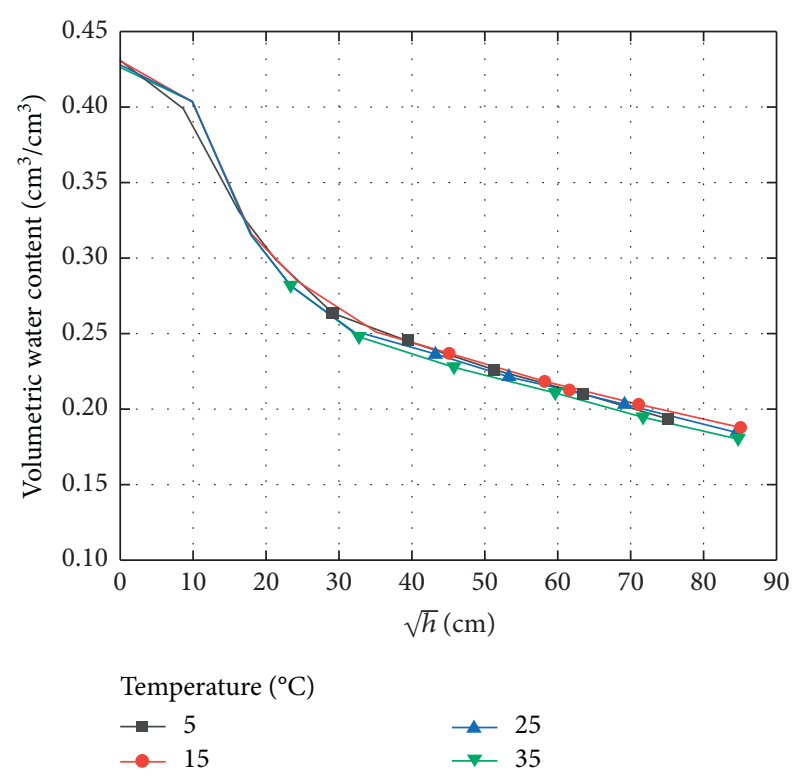

Figure 3: SWCC of loess with $1.5 \mathrm{~g} / \mathrm{cm}^{3}$ dry density at a temperature of $5,15,25$, and $35^{\circ} \mathrm{C}$.

$$
\theta(h)=\theta_{r}+\frac{\theta_{s}-\theta_{r}}{\left[1+|\alpha h|^{n}\right]^{m}}
$$

where $\theta_{r}$ is the residual volumetric liquid water content; $\theta_{s}$ is the quasi-saturated volumetric liquid water content; and $\alpha$, $m$, and $n$ are coefficients; $m=1-(1 / n)$.

The specific water volume of unsaturated loess is $-(\mathrm{d} \theta / \mathrm{d} h)$, obtained by deriving $\theta(h)$ and represented by $C(\theta)$ in the following equation:

$$
C(\theta)=(n-1) \alpha\left(\theta_{s}-\theta_{r}\right)\left(\frac{\theta-\theta_{r}}{\theta_{s}-\theta_{r}}\right)^{(1 / m)}\left[1-\left(\frac{\theta-\theta_{r}}{\theta_{s}-\theta_{r}}\right)^{(1 / m)}\right]^{m} .
$$

The experimental results of four types of density of unsaturated loess were fitted and analyzed. $\theta_{r}, \theta_{s}, \alpha, n$, and $m$ can be written as follows:

$$
\begin{aligned}
& \theta_{r}=-0.38+0.36 \rho_{d}, \\
& \theta_{s}=1-0.38 \rho_{d}, \\
& \alpha=e^{7.11-7.52 \rho_{d}}, \\
& n=0.359+0.414 \rho_{d}+0.222 \rho_{d}^{2}, \\
& m=1-\frac{1}{n} .
\end{aligned}
$$

The specific water capacity of unsaturated loess can be obtained by substituting $\theta_{r}, \theta_{s}, \alpha, n$ and $m$ into equation (4).

Then, regression analysis was carried out on the experimental values of water diffusivity $D(\theta)$ of unsaturated loess with four densities.
The regression equation is

$$
D(\theta)=e^{\left(p_{1} \theta^{2}+p_{2} \theta+p_{3}\right)}
$$

where $p_{1}, p_{2}, p_{3}$ are the parameters varying with density and $\theta$ is the volumetric liquid water content. Regression analysis was carried out on the change of $p_{1}, p_{2}$, and $p_{3}$ with density. The regression equations are shown in the three following equations:

$$
\begin{aligned}
& p_{1}=84.55 \rho_{d}+45.15 \\
& p_{2}=56.00 \rho_{d}+61.76 \\
& p_{3}=5.72 \rho_{d}-12.05 .
\end{aligned}
$$

By substituting $C(\theta)$ and $D(\theta)$, the permeability coefficient of unsaturated loess considering the effect of density was obtained using the following equation:

$$
K\left(\theta, \rho_{d}\right)=C\left(\theta, \rho_{d}\right) D\left(\theta, \rho_{d}\right)
$$

To verify the equation, the parallel method was used in the water diffusion experiments and matrix suction experiments. The calculated results were compared with the measurement results obtained by parallel experiments. The correlation coefficients are shown in Table 2, indicating that equation (10) can be used to fit the experiment results appropriately.

\section{Analysis of Permeability Coefficient Equation}

Equation (10) was obtained based on the results. It was applied to calculate the permeability coefficient of loess with different densities and water contents to explore the change in permeability coefficient of unsaturated loess with density and water content. The calculation results are shown in Figures 5 and 6 . Figure 5 shows the variation of permeability coefficient with the water content of unsaturated loess with different densities. Figure 6 shows the change in permeability coefficient with the density of unsaturated loess at different water contents. The semilog plot was used.

Figure 5 shows that the change in water content of unsaturated loess can lead to several orders of magnitude changes in its permeability coefficient; under the condition of constant dry density, the relationship between permeability coefficient and water content in semilog plot is approximately linear, indicating that the value of permeability coefficient increases monotonously with the increase in water content, similar to the increase in water diffusion coefficient with water content. From the absolute change, when the water content is large, the change in permeability coefficient with water content is relatively large, and when the water content is small, the change in permeability coefficient with water content is relatively small. Because of the use of order coordinate, a wave-type curve appeared in the calculation curve, which reflects that the increased rate of permeability coefficient increases with the increase in water content; i.e., the higher the soil moisture content, the faster the increase in speed. 

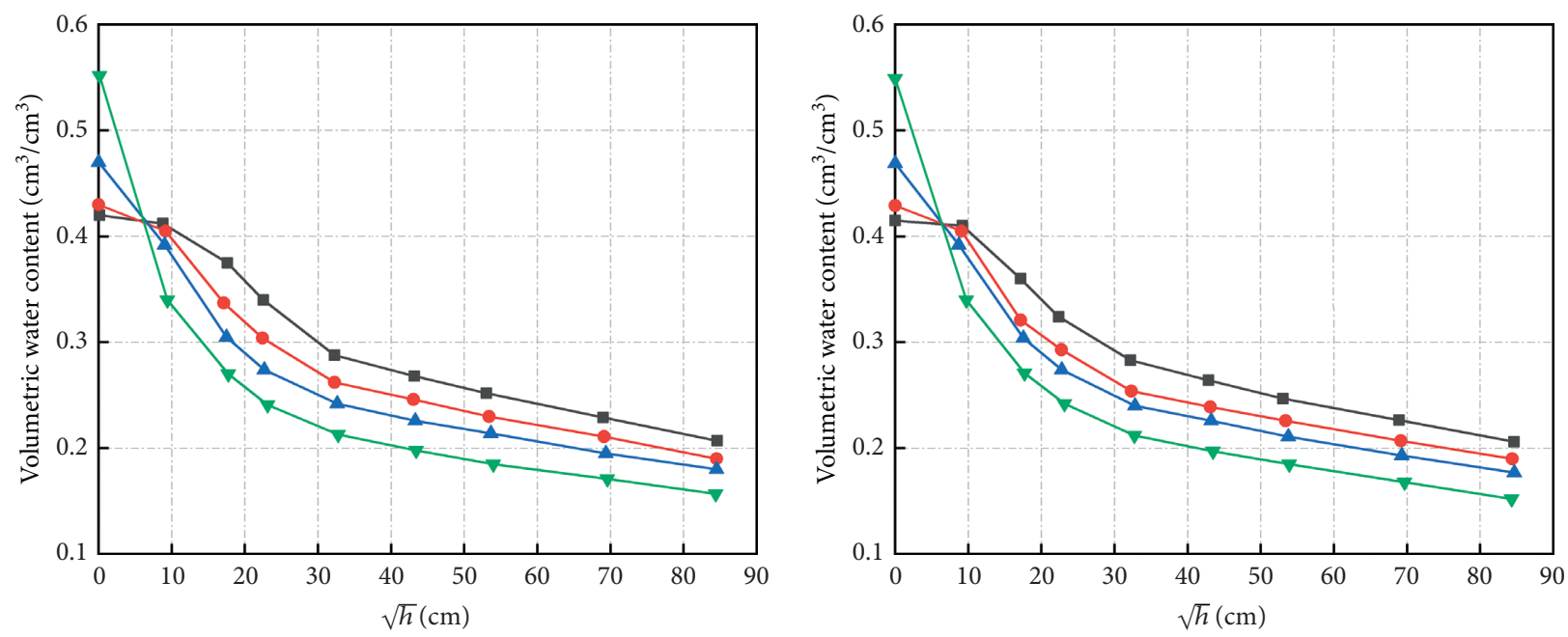

Dry density $\left(\mathrm{g} / \mathrm{cm}^{3}\right)$

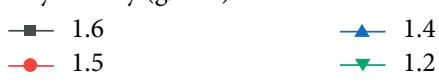

(a)

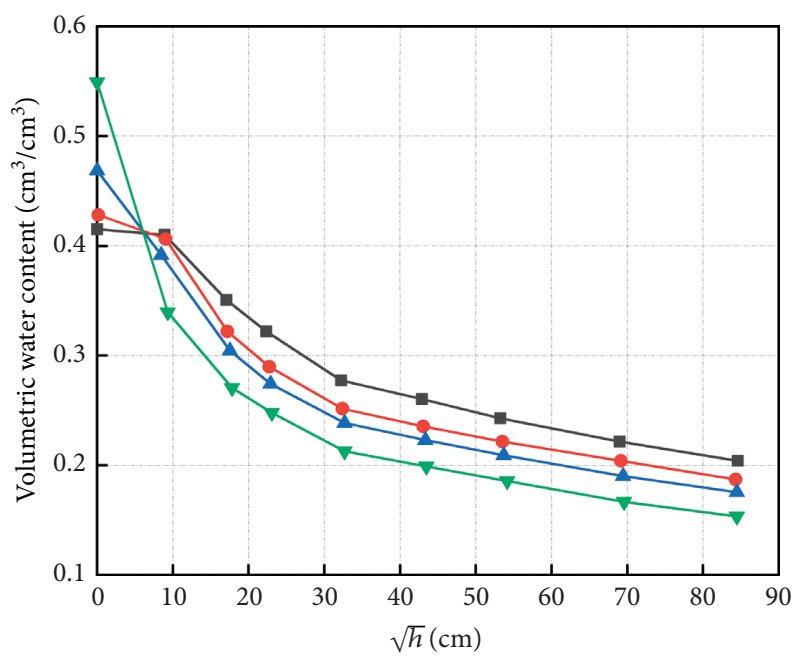

Dry density $\left(\mathrm{g} / \mathrm{cm}^{3}\right)$

$$
\begin{array}{ll}
-1.6 & \rightarrow 1.4 \\
-1.5 & \rightarrow
\end{array}
$$

(c)
Dry density $\left(\mathrm{g} / \mathrm{cm}^{3}\right)$

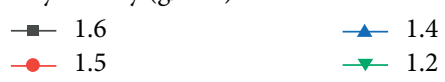

(b)

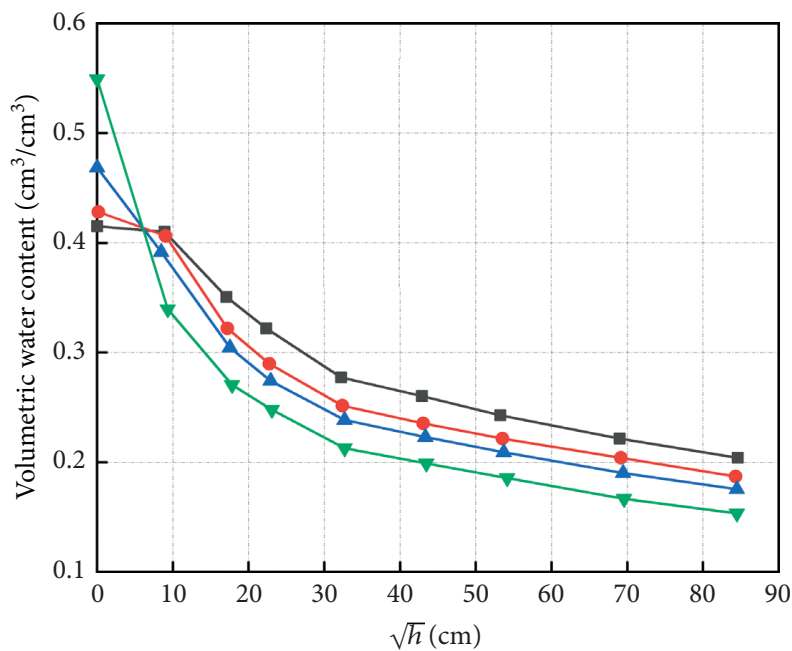

Dry density $\left(\mathrm{g} / \mathrm{cm}^{3}\right)$

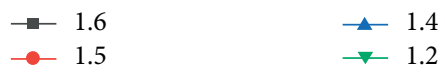

(d)

Figure 4: SWCC of loess with dry density of $1.2,1.4,1.5$, and $1.6 \mathrm{~g} / \mathrm{cm}^{3}$ at a temperature of (a) 5 , (b) 15 , (c) 25 , and (d) 35 .

TABLE 2: Correlation coefficients.

\begin{tabular}{lc}
\hline$\rho_{d}\left(\mathrm{~g} / \mathrm{cm}^{3}\right)$ & Correlation coefficient \\
\hline 1.2 & 0.979 \\
1.4 & 0.985 \\
1.5 & 0.976 \\
1.6 & 0.975 \\
\hline
\end{tabular}

Figure 6 shows that when the soil density is small, the change in permeability coefficient with the density of unsaturated loess at different water contents is small. When the soil density is relatively large, the change in permeability coefficient with the density of unsaturated loess at different water contents is relatively large. The permeability coefficient of dense loess is sensitive to the change in density.

\section{Water Migration of Unsaturated Loess}

To apply the obtained permeability parameters to the calculation of water migration, a one-dimensional (1D) water transfer model of unsaturated loess was established. The obtained permeability parameters were substituted into the model to obtain the calculated values of water migration. According to the measured results of water migration of unsaturated loess, the calculated values of water migration 


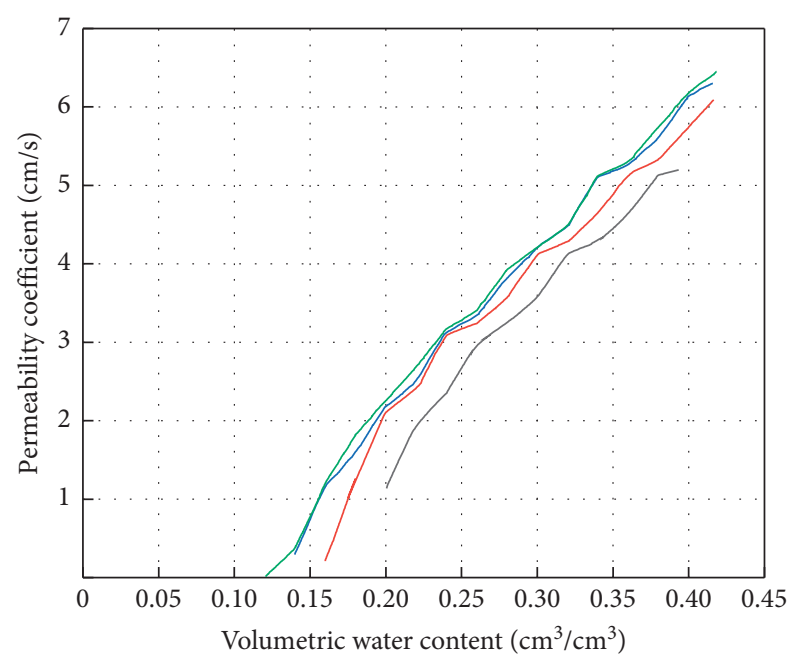

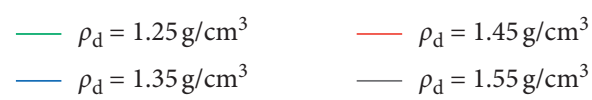

FIgURE 5: Curve of permeability coefficient with volumetric liquid water content of loess with dry densities of 1.25, 1.35, 1.45, and $1.55 \mathrm{~g} / \mathrm{cm}^{3}$.

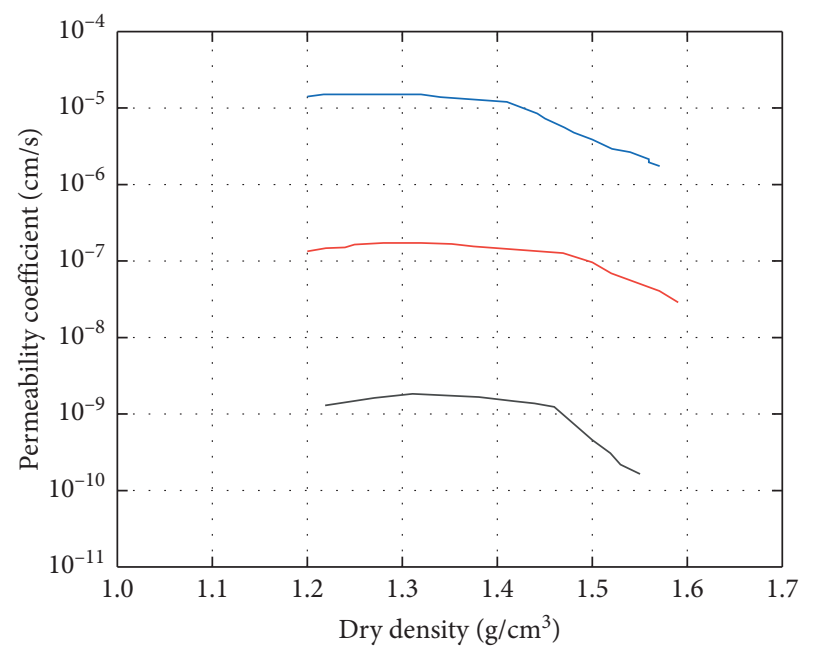

Volumetric water content $\left(\mathrm{cm}^{3} / \mathrm{cm}^{3}\right)$

$-0.40$

$-0.30$

$-0.20$

FIGURE 6: Curve of permeability coefficient with dry density of loess with volumetric liquid water contents of $0.40,0.30$, and $0.20 \mathrm{~cm}^{3}$ / $\mathrm{cm}^{3}$.

based on the permeability parameters of loess were compared with the measurement values of water migration experiments. The comparison results can be used to verify the diffusion coefficient and SWCC of loess measured above.

6.1. Experimental Setup for Water Migration. Experiments were conducted on the water migration of unsaturated loess, and the results of water migration were obtained. First, Arid soil (with a small water content) and wet soil (with a large water content) were prepared and then placed in a PVC pipe with a length of $10 \mathrm{~cm}$ and sealed at one end. Then, they were docked in pairs. Figure 7 shows the experiment apparatus of water migration, and Table 3 shows the physical properties of loess samples.

6.2. Theoretical Model for Gaseous Water Migration. The gaseous water present in loess is mainly transported by the diffusion of saturated gaseous water. Matrix suction makes pore water form a concave meniscus, affecting the saturated gaseous water pressure of gaseous water migration and diffusion. According to the law of conservation of mass and Fick's first law, a 1D diffusion equation of gaseous water in unsaturated loess equations (11) and (12) can be obtained.

$$
\begin{aligned}
\rho_{\mathrm{d}} \frac{\partial w_{a}}{\partial t} & =\frac{\partial J_{\mathrm{x}}}{\partial x}, \\
J_{x} & =-D_{x} \frac{(1-S) n}{R_{v} T} \frac{\partial P_{v 0}}{\partial x},
\end{aligned}
$$

where $w_{a}$ is the moisture content of gaseous water; $J_{x}$ is the water air flow rate in $x$ direction; $D_{x}$ is the diffusion coefficient of gaseous water in $x$ direction; $S$ is the saturation of soil; $n$ is the porosity; $R_{v}$ is the gaseous water constant with a value of $0.4615(\mathrm{KJ} /(\mathrm{kg} \cdot \mathrm{K}))$; $\mathrm{T}$ is the absolute temperature; $P_{v 0}$ is the gaseous water pressure in the pore in the saturated state, where the value is related to the temperature and matric suction of soil.

Considering the relationship between matric suction and $P_{v 0}, P_{v 0}$ can be expressed using Kelvin equation in the two following equations:

$$
\begin{aligned}
& \frac{2^{e}}{r}=-\frac{\rho_{w}}{\rho_{v 0}} P_{v 0} \ln \frac{P_{v}}{P_{v 0}}, \\
& \rho_{v 0}=\frac{P_{v 0}}{R_{v 0} T},
\end{aligned}
$$

where $P_{v}$ is the saturated gaseous water pressure on free surface; $e$ is the surface tension of curved liquid surface, which is determined according to the SWCC obtained in this study; $r$ is the radius of curvature; $\rho_{v 0}$ is the density of gaseous water; $\rho_{w}$ is the density of water.

In unsaturated soils, the matric suction $u_{a}-u_{w}$ causes the interface between gaseous water and liquid water to bend, and it can be obtained using the following equation:

$$
-\frac{2^{e}}{r}=u_{a}-u_{w} \text {. }
$$

By combining equations (13) and (15), $P_{v 0}$ can be represented as follows:

$$
P_{v 0}=P_{v} \exp \left[-\frac{u_{a}-u_{w}}{\rho_{w} R_{v} T}\right] .
$$

$P_{v}$ is also affected by temperature and can be represented as follows: 


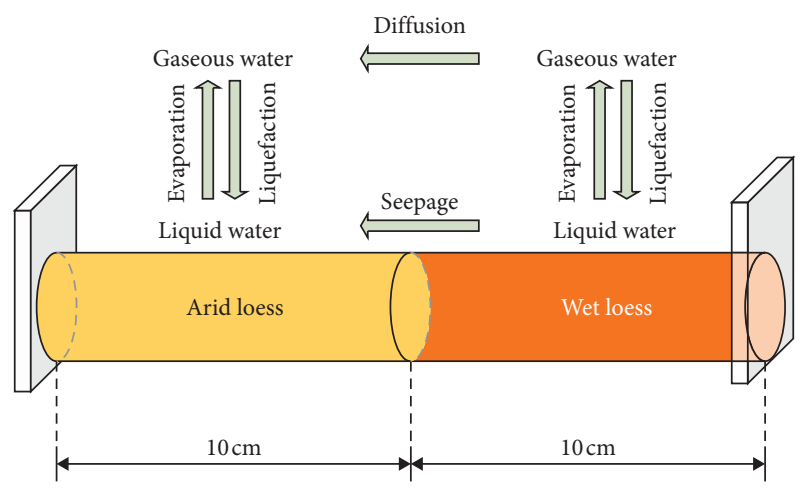

FIgURE 7: Test apparatus for water migration.

$$
P_{v}=B \exp \left(-\frac{F}{R_{v} T}\right),
$$

where $B$ and $F$ are constants with values of $2.315 \times 10^{8} \mathrm{kPa}$ and $2489.3(\mathrm{~kJ} / \mathrm{kg})$, respectively; $u_{a}-u_{w}$ is the matric suction of unsaturated soil.

By combining equations (16) and (17), $P_{v 0}$ can be represented as follows:

$$
P_{v 0}=B \cdot \exp \left(-\frac{u_{a}-u_{w}}{d_{w}-R_{v} T}-\frac{F}{R_{v} T}\right) .
$$

$D_{x}$ can be obtained from the following formula:

$$
\begin{aligned}
& D_{x}=D_{v} \frac{\partial \rho}{\partial \theta_{v}}, \\
& D_{v}=D_{a} \alpha\left(\frac{\varepsilon-\theta}{\rho_{w}}\right),
\end{aligned}
$$

where $D_{v}$ is the gaseous water diffusion coefficient $\left(\mathrm{cm}^{2} / \mathrm{s}\right)$ with the reference system of aerated pores in soil $\left(D_{x}\right.$ taking the soil as the reference system); $D_{a}$ is the gaseous water diffusion coefficient in still air $\left(\mathrm{cm}^{2} / \mathrm{s}\right)$, and the value of $D_{a}$ is $2.12 \times 10^{-5}(T / 273.15)^{2}$, a constant in this study; $a$ is the curvature, 0.66 in this study; $\theta_{v}$ is the volume moisture content $\left(\mathrm{cm}^{3} / \mathrm{cm}^{3}\right) ; \eta$ is the porosity (\%); and $\rho$ is the saturated density of gaseous water, which is related to temperature.

\subsection{Theoretical Model for Liquid Water Migration. Liquid} water migration conforms to Darcy's law. The liquid water migration in a loess area is unsteady, and the water diffusion rate and matrix suction affect the permeability coefficient. Based on Darcy's law and law of conservation of mass, a 1D equation of liquid water transport in unsaturated loess can be obtained.

$$
\frac{\partial \theta_{L}}{\partial t}=\frac{\partial}{\partial x}\left[K\left(\theta_{L}\right) \cdot \frac{\partial \psi_{m}}{\partial x}\right],
$$

where $\theta_{L}$ is the volumetric liquid water content of liquid water, which should be converted into the mass water content of liquid water in calculation. $K$ is the permeability coefficient of liquid water in the direction of seepage, which has been measured in this study; and $\psi_{m}$ is the matrix potential of liquid water, which was obtained from the SWCC measured in this study.

By combining equations (11) and (20), a governing equation for gaseous and liquid water flow can be obtained using the following mass conservation equation:

$$
\frac{\partial \theta}{\partial t}=\frac{\partial}{\partial x}\left[K\left(\theta_{L}\right) \cdot \frac{\partial \psi_{m}}{\partial x}\right]+\frac{\partial J_{x}}{\partial x},
$$

where $\theta$ is the mixed volumetric water content, namely, the sum of liquid and gaseous water contents.

6.4. Boundary and Initial Conditions. The boundary conditions are presented using the following equation:

$$
\frac{\partial w(0, T)}{\partial x}=\frac{\partial w(l, T)}{\partial x}=0
$$

The initial conditions were obtained using the following equation:

$$
w(x, 0)= \begin{cases}7.2 \%, & 0 \leq x \leq \frac{l}{2}, \\ 14.2 \%, & \frac{l}{2}<x \leq l,\end{cases}
$$

where $w$ is the mass water content $\left(w_{l}+w_{a}\right)(\%)$ and $l$ is the distance from the arid end $(\mathrm{cm})$.

6.5. Calculation of Water Migration. The finite element equations of liquid water and gaseous water migration were transformed using a backward difference scheme. The boundary conditions are consistent with the boundary conditions of water transfer experiment. Combined with the model parameters obtained by the author, the distribution laws of gaseous and liquid water at different migration times were iterated. Considering the interaction of gaseous water migration and liquid water migration, the migration amounts of gaseous water and liquid water in the $i$-th time step were calculated simultaneously. After the iteration converges to the optimal value, the gaseous water content and liquid water content were added to obtain the total water content. Using the total water content of the $i$-th time step, the migration amounts of gaseous water and liquid water in the $i+1$-th time step can be calculated. In this manner, the results of gas-liquid mixed water migration can be obtained under a predetermined water transport time. The calculation process is shown in Figure 8. To determine the accuracy of calculation results, $\mathrm{d} t$ and $\mathrm{d} s$ were set as $2.4 \mathrm{~s}$ and $0.001 \mathrm{~m}$, respectively, by trial calculation. The calculation program was compiled by FORTRAN.

6.6. Comparison of Calculated and Experimental Results. The experiment soil samples with a length of $200 \mathrm{~mm}$ were divided into 100 sections. The step length $\mathrm{d} x$ is $2 \mathrm{~mm}$, and the time step dt is $2.4 \mathrm{~s}$. The experiment was carried out under different conditions. Figure 9 shows that the 
TABLE 3: Physical properties of loess samples for water migration experiment.

\begin{tabular}{lcccccc}
\hline $\begin{array}{l}\text { Plastic } \\
\text { limit }\end{array}$ & $\begin{array}{c}\text { Liquid } \\
\text { limit }\end{array}$ & $\begin{array}{c}\text { Specific } \\
\text { gravity }\end{array}$ & $\begin{array}{c}\text { Maximum arid density } \\
\left(\mathrm{g} / \mathrm{cm}^{3}\right)\end{array}$ & $\begin{array}{c}\text { Dry density }(\mathrm{g} / \\
\left.\mathrm{cm}^{3}\right)\end{array}$ & $\begin{array}{c}\text { Mass water content of arid } \\
\text { loess }(\%)\end{array}$ & $\begin{array}{c}\text { Mass water content of wet } \\
\text { loess }(\%)\end{array}$ \\
\hline 18.1 & 29.6 & 2.70 & 1.69 & 1.35 & 7.2 & 14.2 \\
\hline
\end{tabular}

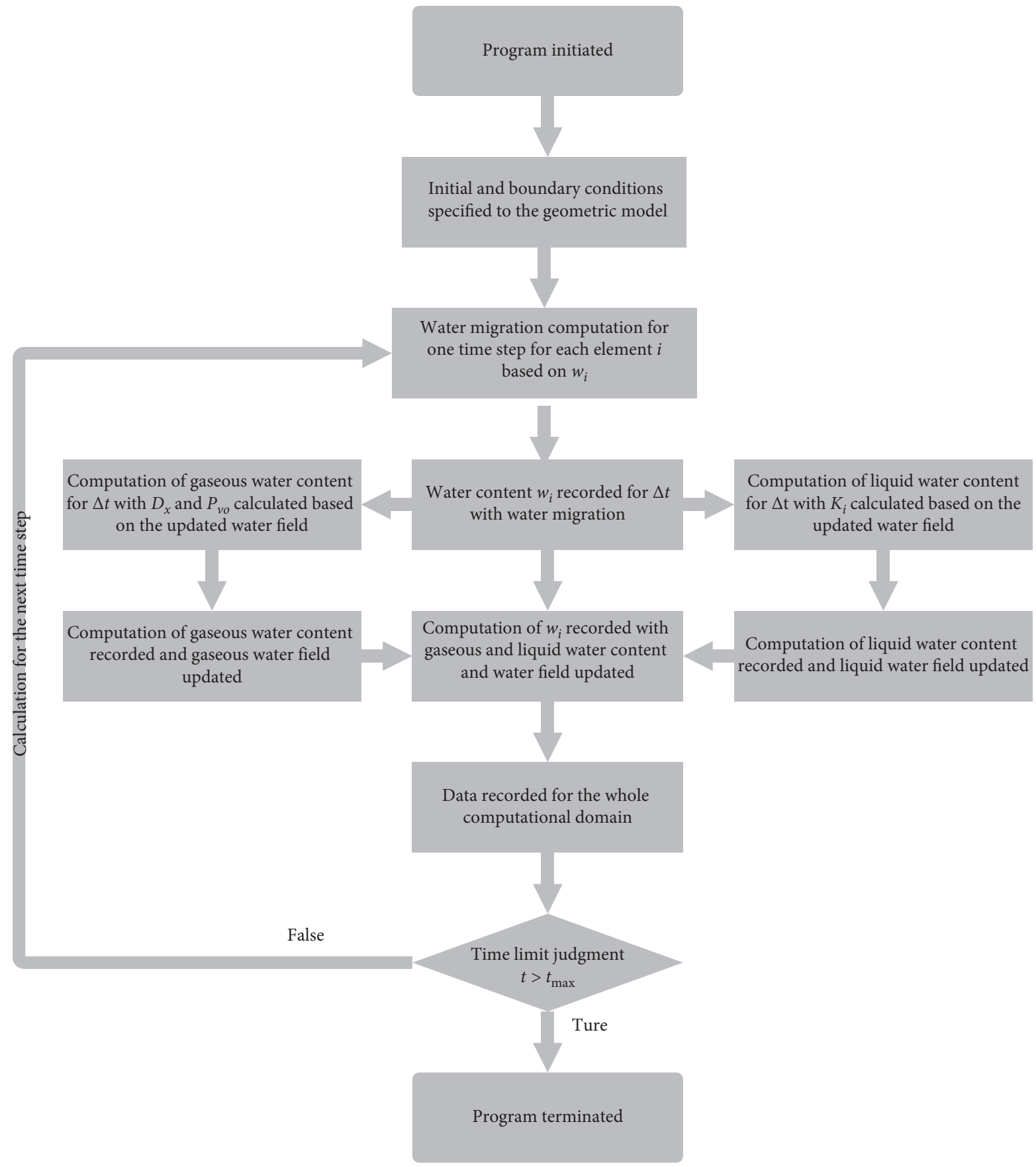

Figure 8: Computational procedure.

correlation coefficients between fitted and measured mass water contents after days 8,12 , and 16 of water migration are not less than 0.98 , indicating that it is reliable to calculate the water migration of unsaturated loess by applying the permeability parameters obtained in the experiment in this study. In the contact area of arid and wet soil samples, the change in calculated water content is obviously greater than the results. Apart from the contact area, the calculated water 


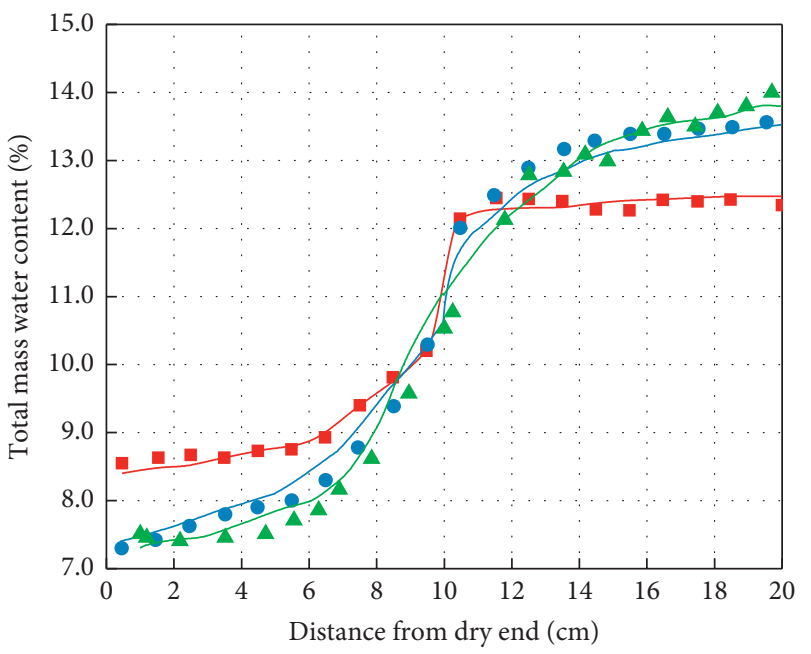

$\begin{array}{ll}\text { Fitted by water migration model } & \text { Measured data } \\ \text { Days: } & \text { Days: } \\ 16 & 16 \\ 12 & -12 \\ 8 & \text { - } 8\end{array}$

Figure 9: Fitted and measured mass water contents after days 8, 12, 16, and 20 of water migration.

content is close to the experimental value, but the change in calculated water content is still greater. This is mainly because the model calculation ignores the hysteresis effect of water migration, and the contact area of arid and wet soil samples has a moisture content gradient, where the water migration is intense, and the hysteresis effect of water migration is obvious. The hysteresis effect is most obvious in the initial stage of water migration, but, with the increase in migration time, the hysteresis effect gradually weakens, and the fitting value and measured value converge. On the whole, the water content distribution obtained by the coupling calculation of water transfer between gas and liquid with the measured permeability parameters in this study is consistent with the obtained water content distribution in the experiment.

Figure 9 shows that water migration flowing from wet soil to arid soil results in significant redistribution of water between arid and wet soils. The change in water content can be divided into three stages according to the time: In the first stage (days $0-8$ ), the water content of loess near the arid wet contact surface changes significantly, but the change in soil moisture content far away from the contact surface is not obvious. In the second stage (days 8-16), the water content of loess near the arid wet contact surface remains stable, and the water content far away from the contact surface changes. In the third stage (after day 16), the stable stage of water migration is reached, and the water content in different places does not change. In addition, the change in water between arid and wet soils is antisymmetric from the arid wet contact surface. To facilitate the analysis, the arid soil (water diversion end) was taken for analysis. In the first stage (days 0-8), the water content of right side of arid soil increases rapidly. The closer the distance between the arid and wet contact surfaces, the greater the increase in water content. However, the water content of left side of arid soil (far away from the arid wet contact surface) changes slightly. This is mainly because, in the early stage of water migration, only the water content gradient near the arid wet interface is used as the driving force, while the moisture content in the soil is uniform, and no moisture gradient acts as the driving force. Therefore, the moisture of wet soil flows into the right end of arid soil from the contact surface and accumulates at the right end. With water accumulation in the right end, a moisture content gradient is formed on the left and right sides of arid soil, and the water migration enters the second stage (days 8-16). Driven by the gradient, the water present on the right side moves to the left side, and the water content of the left side soil increases rapidly. However, because of the water supply from the wet soil on the right side, the water content of right side remains unchanged and does not decrease. With the increase in water content in the right end, the water contents at the left and right ends gradually become equal. With the disappearance of driving force, the water migration enters a stable stage, and the water migration enters the third stage (after day 16).

Figure 10 shows that gaseous water migration is the main water migration in total water migration. According to the water migration experiment on the 8th day, the gaseous water migration accounts for about $70 \%$ of total migration. On the 12th day of water migration experiment, the proportion of gaseous water migration decreased but still accounted for $65 \%$ of total migration. On the 16 th day of water migration experiment, the proportion of gaseous water migration further decreased, accounting for about $50 \%$ of total migration. Thus, the migration of gaseous water was faster than that of liquid water. With the increase in time, the migration of gaseous water weakened, and the migration of liquid water increased. However, in general, the 


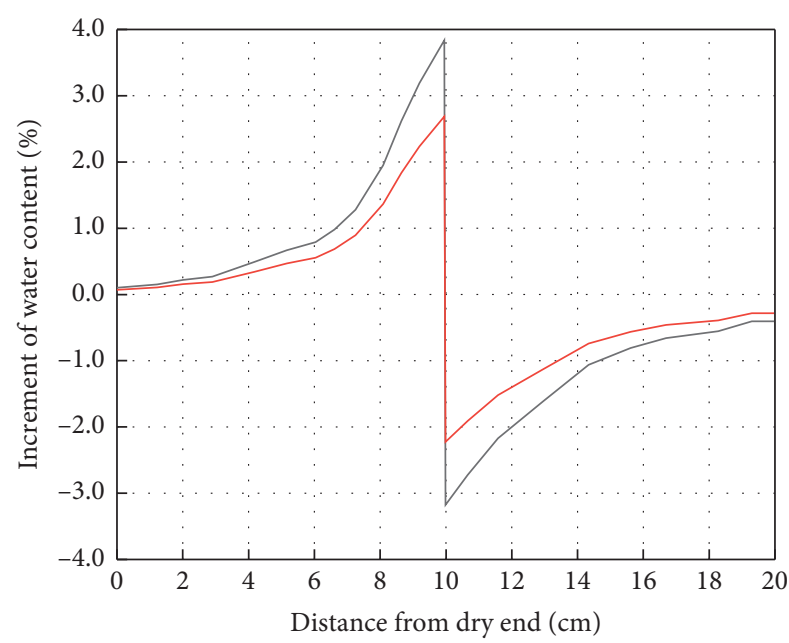

Fitted values of days 8 :

_ Total water content

_ Gaseous water content

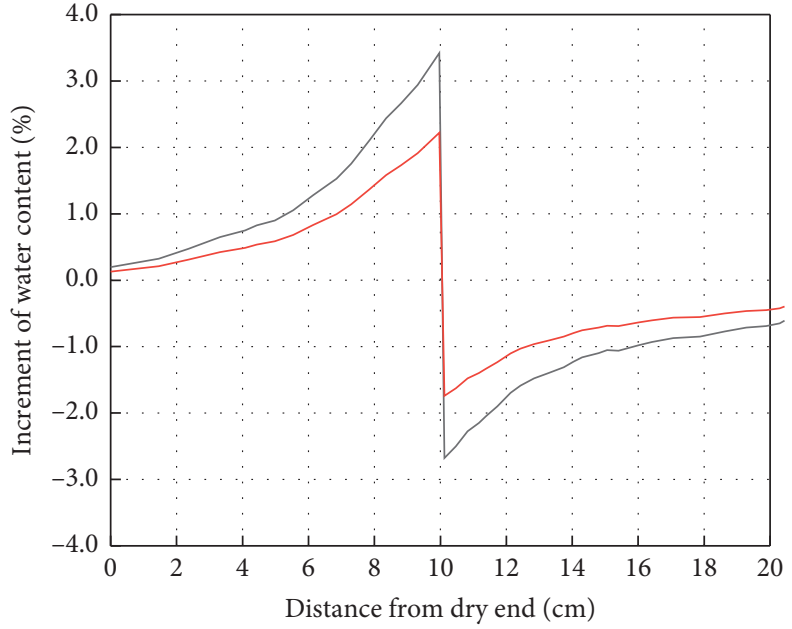

Fitted values of days 12:

- Total water content

_ Gaseous water content

(a)

(b)

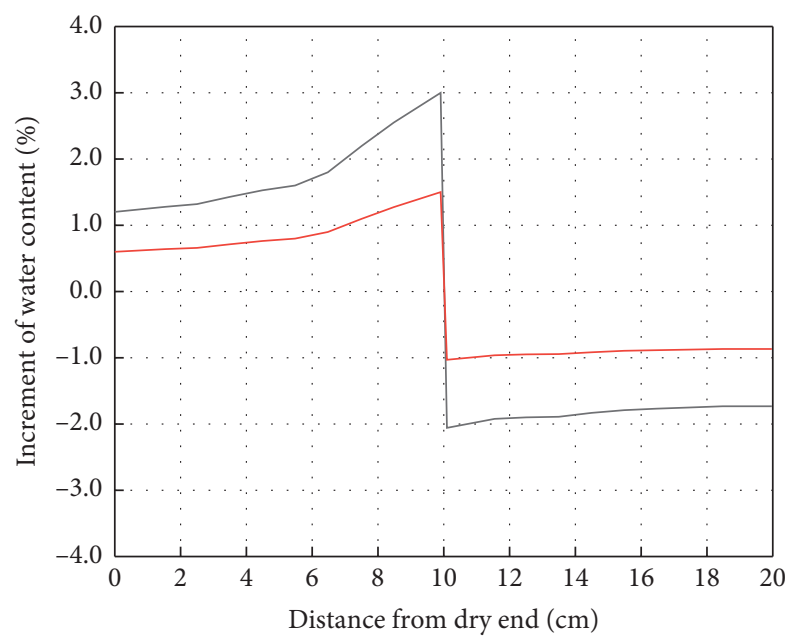

Fitted values of days 16 :

Total water content

_ Gaseous water content

(c)

FIGURE 10: Fitted values of increase in total gaseous water content of water migration after days (a) 8, (b) 12, and (c) 16.

migration of gaseous water accounts for the main part of total migration.

\section{Conclusion}

(1) The relationship between water diffusivity and water content of loess with different densities was obtained using horizontal soil column infiltration method, and the SWCC of loess with different densities was obtained using a high-speed centrifuge method. The experiment results show that when the water content is large, the effect of density on the diffusion rate and matric suction of loess is very significant.
(2) A method was developed to determine the permeability coefficient of unsaturated loess considering the effect of density by regression analysis. The variation trend of permeability coefficient of unsaturated loess with density and water content was analyzed. The permeability coefficient increases monotonically with the increase in water content, and the permeability coefficient of dense loess is sensitive to the change in density.

(3) Considering the characteristics of mixed migration of gas and liquid water in unsaturated loess, an equation was derived for gas-liquid water transfer in 
unsaturated loess. The water diffusivity and SWCC of loess obtained by experiment were substituted into the equation for calculation. The calculated results are consistent with the experimental results of water migration experiment. This shows that the model of water migration established in this study can be used to calculate the water migration of unsaturated loess, and it is reasonable to apply the measured water diffusivity and SWCC in this study to the calculation of water migration.

\section{Data Availability}

The data used to support the findings of this study are available from the corresponding author upon request.

\section{Conflicts of Interest}

The authors declare that they have no conflicts of interest.

\section{Acknowledgments}

This research was financially supported by the Key Research and Development Program of Shaanxi (no. 2018ZDCXL-SF30-9) and the Key Projects of Natural Science Basic Research in Shaanxi Province (no. 2020ZJ-49).

\section{References}

[1] S. Jeong, Y. Kim, H. Park, and J. Kim, "Effects of rainfall infiltration and hysteresis on the settlement of shallow foundations in unsaturated soil," Environmental Earth Sciences, vol. 77, no. 13, 2018.

[2] E. L. Lemoubou, H. T. Tagne Kamdem, J. R. Bogning, and E. H. Zefack Tonnang, "Thermal, moisture, and solute transport responses effects on unsaturated soil hydraulic parameters estimation," Water Resources Research, vol. 55, no. 12, 2019.

[3] F. Zhang, G. W. Wilson, and D. G. Fredlund, "Permeability function for oil sands tailings undergoing volume change during drying," Canadian Geotechnical Journal, vol. 55, no. 2, pp. 191-207, 2018.

[4] R. Kaewsong, C. Zhou, and C. W. W. Ng, "Modelling effects of recent suction history on small-strain stiffness of unsaturated soil," Canadian Geotechnical Journal, vol. 56, no. 4, 2019.

[5] K. Tian, A. Yang, K. Nie, H. Zhang, J. Xu, and X. Wang, "Experimental study of steady seepage in unsaturated loess soil," Acta Geotechnica, vol. 15, no. 9, 2020.

[6] H. Trabelsi, B. Hadrich, and H. Guiras, "Evaporation, shrinkage and intrinsic permeability of unsaturated clayey soil: analytical modelling versus experimental data," Arabian Journal of Geosciences, vol. 11, no. 8, 2018.

[7] J. Sun, "Permeability of particle soils under soil pressure," Transport in Porous Media, vol. 123, no. 2, pp. 257-270, 2018.

[8] Q. Jin, X. Cui, J. Su, T. Lu, J. Wang, and R. Han, "Laboratory measurement and analysis of the deteriorated layer permeability coefficient of soil-cement deteriorated in a saline environment," Materials, vol. 12, no. 14, 2019.

[9] S. Nazari, M. Hassanlourad, E. Chavoshi, and A. Mirzaii, "Experimental investigation of unsaturated silt-sand soil permeability," Advances in Civil Engineering, vol. 2018, pp. 1-12, 2018.
[10] H. Ke, P. Li, Z. Li, P. Shi, and J. Hou, "Soil water movement changes associated with revegetation on the loess plateau of China," Water, vol. 11, no. 4, 2019.

[11] J. Qiao, Y. Zhu, X. Jia, L. Huang, and M. A. Shao, "Development of pedotransfer functions for soil hydraulic properties in the critical zone on the Loess Plateau, China," Hydrological Processes, vol. 32, no. 18, 2018.

[12] J. Xu, Y. Li, S. Wang, Q. Wang, and J. Ding, "Shear strength and mesoscopic character of undisturbed loess with sodium sulfate after dry-wet cycling," Bulletin of Engineering Geology and the Environment, vol. 79, no. 3, 2019.

[13] L. Zhang, S. Qi, L. Ma et al., "Three-dimensional pore characterization of intact loess and compacted loess with micron scale computed tomography and mercury intrusion porosimetry," Scientific Reports, vol. 10, no. 1, 2020.

[14] Y.-n. Wei, W. Fan, N. Yu, L.-s. Deng, and T. Wei, "Permeability of loess from the South Jingyang Plateau under different consolidation pressures in terms of the threedimensional microstructure," Bulletin of Engineering Geology and the Environment, vol. 79, no. 9, 2020.

[15] H. Wang, H. Qian, and Y. Gao, "Non-darcian flow in loess at low hydraulic gradient," Engineering Geology, vol. 267, 2020.

[16] L. Wang, C. Li, J. Qiu, K. Wang, T. Liu, and H. Li, “Treatment and effect of loess metro tunnel under surrounding pressure and water immersion environment," Geofluids, vol. 2020, pp. 1-18, 2020.

[17] J. Liu, X. a. Li, Q. Xue, and Z. Guo, "Experimental study on air permeability and microscopic mechanism of intact and remolded Malan loess, Loess Plateau, China," Bulletin of Engineering Geology and the Environment, vol. 79, no. 8, 2020.

[18] G. Lin, W. Chen, P. Liu, and W. Liu, "Experimental study of water and salt migration in unsaturated loess," Hydrogeology Journal, vol. 27, no. 1, 2018.

[19] K. Hou, H. Qian, Q. Zhang et al., "Influence of Quaternary paleoclimate change on the permeability of the loess-paleosol sequence in the Loess Plateau, northern China," Earth Surface Processes and Landforms, vol. 45, no. 4, 2020.

[20] J. Wang, D. Zhang, C. Chen, and S. Wang, "Measurement and modelling of stress-dependent water permeability of collapsible loess in China," Engineering Geology, vol. 266, 2020.

[21] J. Xu, W. Lan, Y. Li, S. Wang, W.-C. Cheng, and X. Yao, "Heat, water and solute transfer in saline loess under uniaxial freezing condition," Computers and Geotechnics, vol. 118, 2020.

[22] W. R. Gardner and M. Fireman, "Laboratory studies of evaporation from soil columns in the presence of a water table," Soil Science, vol. 85, no. 5, pp. 244-249, 1958.

[23] M. T. Van Genuchten, "A closed-form equation for predicting the hydraulic conductivity of unsaturated soils," Soil Science Society of America Journal, vol. 44, no. 5, 1980.

[24] D. G. Fredlund and A. Xing, "Equations for the soil-water characteristic curve," Canadian Geotechnical Journal, vol. 31, no. 4, pp. 521-532, 1994. 